\title{
MULTI-CENTRIC, DOUBLE BLIND STUDY OF EMAMI BLENDED EDIBLE VEGETABLE OIL (EBEVO1) ON IMMUNITY
}

\begin{tabular}{|c|c|}
\hline Ayurveda & \\
\hline Dr. Banani Das* & AMS, MD (Ay.), Ayur Jyoti Clinic, Krishnapur, Kolkata- 700101. *Corresponding Author \\
\hline $\begin{array}{l}\text { Dr. Abhay Shankar } \\
\text { Ojha }\end{array}$ & , LCMC, AYU Clinic, 3/1 M. M. Feeder Road, Belgharia, Kolkata -700056. \\
\hline $\begin{array}{l}\text { Dr. Pariksh } \\
\text { Debnath }\end{array}$ & , \\
\hline
\end{tabular}

\begin{abstract}
Background: The composition of Emami Blended Edible Vegetable Oil (EBEVO1) is refined soyabean oil (75\% by wt) and refined rice bran oil ( $25 \%$ by wt) with add on vitamins such as A, C, D, E \& omega 3 fatty acids in order to enrich its nutritional value. Vit. A is involved in the development of the immune system and plays regulatory roles in cellular immune responses and humoral immune processes. Vit. A has demonstrated a therapeutic effect in the treatment of various infectious diseases. Crucial immune organs need constant dietary intake to maintain Vit. A concentration. Vitamin C is important in all stressful conditions that are linked to inflammatory processes and involve immunity.

Study objectives: The sponsor, M/s Emami Agrotech Pvt. Ltd., developed the Emami Blended Edible Vegetable Oil (EBEVO1) which was evaluated upon the user acceptability and efficacy parameters. The baseline data of the evaluating parameters was compared with the results obtained by the end of the study (90days).

Trial Design: The study was a randomized, multicentric, double-blind and controlled. The clinical trial protocol, patient information sheets, and consent forms were approved by the Ethical Committee of the Independent Research Ethics Society. The study was conducted after receiving the consent from the Ethics Committee (March 15, 2019). The Investigators have strictly followed the Standard Operating Procedures (SOPs) for the study following the Good Clinical Practice (GCP) and adhered to the Declaration of Helsinki. He/she thoroughly examined the study participants in his/her specific centre(s).

Result: The results are encouraging where Group - A Oil shows better results than Group - B Oil due to value addition through Vitamins.

Conclusion: Emami Blended Edible Vegetable Oil contains 5 essential nutrients such as vitamin A, C, D, E and Omega 3 that have already been clinically confirmed in numerous trials around the world. The special blend of added nutrients that enhance the metabolic functions to enhance immunity to prevent minor general disorders or infections.
\end{abstract}

\section{KEYWORDS}

Emami Blended Edible Vegetable Oil, Improvement in stamina, Immunity

\section{INTRODUCTION}

Numerous micronutrients naturally abundant in oilseeds prevent the risk of cardiovascular diseases by reducing hyperlipidemia and oxidative stress. Emami Agrotech Pvt. Ltd., Kolkata have developed Emami Blended Edible Vegetable Oil (EBEVO1). This blend is a mixture of two refined oils i.e. Refined Soybean oil (75\%) (where omega 3 fatty acid is naturally available) and Refined Rice bran oil $(25 \%)$ with addition of vitamin A, D, C, E. Vitamin A, D, C, E and Omega 3 Fatty Acid are proven to boost immune system and develop humoral immunity. Scientists of Emami Agrotech Pvt. Ltd .conceptualize a hypothesis for the following benefits on the basis of pharmacological actions by added nutrients:

- Vitamin A-This particular vitamin is involved in the development of the immune system and plays regulatory role in cellular immune response and humoral immune responses as well as in the treatment of several infectious disease. ${ }^{1}$

- Vitamin D-Vitamin D plays a crucial role in protecting bones, healing bone fragility. Thus it is very useful for patients with osteoporosis.

- Vitamin $\mathrm{C}-$ Vitamin $\mathrm{C}$ is rich in antioxidant decreasing stress level, preventing high cholesterol and acts as immune boosters.

- Vitamin E - This is also an antioxidant which reduces the generation of free radicals and reactive oxygen species. Hence elevating stamina and reducing oxidative stress.

- Omega-3 fatty acids are essential nutrients that are important in preventing and managing heart disease. ${ }^{4}$

The crude rice bran oil is mainly composed of glycerides $(80 \%)$ while phospholipids, glycolipids, free fatty acids and waxes are also present in less quantity. The RBO is rich source of anti-oxidants especially phytochemical oryzanol, tocopherols and tocotrienols which acts on higher oxidative stability and longer shelf life than other edible oils.

Rice bran Oil is safe for consumption and is being used in various types of cooking and frying.

Soybean oil provides calories, the essential fatty acids and vitamin A and $\mathrm{E}$, but contributes insignificant amounts of Vitamins D and $\mathrm{K}$. It has one of the highest iodine values of oil seeds (a value of 134), which is similar to that of sunflower oil; peanut butter, maize and palm oils have iodine values of 101, 127 and 51, respectively, indicating lower unsaturation compared to SB oil ${ }^{5}$.

Vitamin A is known as an anti- inflammatory vitamin because of its critical role in enhancing immune function. Vitamin A is involved in the development of the immune system and plays regulatory roles in cellular immune responses and humoral immune processes. Vitamin A has demonstrated a therapeutic effect in the treatment of various infectious diseases. Vitamin A leads to a defect in both $\mathrm{T}$ cell-mediated and antibody-dependent immune responses. Vitamin D acts as important stimulant for innate immunity, enhances the antimicrobial effects of macrophages and monocytes, which are important effector cells, fighting against pathogens. Vitamin D on human adaptive immune cells demonstrated an expression of the nuclear VDR as well as vitamin D- activating enzymes in both T- and B cells. ${ }^{6}$ Immunomodulatory effects of vitamin $\mathrm{E}$ have been observed in animal and human models under normal and disease conditions. With advances in understating of the development, function and regulation of dendritic cells (DCs), macrophages, natural killer (NK) cells, T cells, and B cells, recent studies have focused on vitamin E's effects on specific immune cells.

To study the Emami Blended Edible Vegetable Oil Maintenance or improvement in stamina and immunity.

\section{METHODS}

\section{SUBJECT SELECTION CRITERIA}

INCLUSION CRITERIA

- Male and female subjects in the age group of 18 years and above.

- Healthy volunteers and/or volunteers from any one of the diseases, cardio-metabolic syndrome, hypertension, diabetes, hyper acidity and weak in bone health, stamina and immunity.

- Subjects who gave the written informed consent form.

- Participants were fit and no need of any hospitalization.

- Subjects who were willing to cook with the supplied oil.

- Agree to follow the instructions of do's and don'ts in daily life 
(usual physical activity, exercise, usual diet and usual regular lifestyle).

- Study participants willing to perform all the study related procedures including the use of the study oil, allow the physical and other tests and willing to document symptoms and medication.

- Study participants $\{$ and/or legally accepted representative (LAR) \} willing to give the written informed consent form ability to adhere to dosing, visit schedules and meet the study requirements.

\section{EXCLUSION CRITERIA}

- Volunteers/study participants unlikely to comply with the protocol or unable to understand the nature, scope and possible consequences of the study.

- Inability to carry out the necessary testing for the study.

- Hospitalization

- Known HIV-positive, Hepatitis B or C status.

- Volunteers/study participants did not comply with the protocol or unable to understand the nature, scope and possible consequences of the study.

- Female participants conceive before or during the study duration.

\section{DATAANALYSIS}

Categorical data between the baseline \& post treatment was compared with Wilcoxon and Mann Whitney U test. Continuous data was analyzed by Paired Samples t-Test and Independent Samples t-Test. Further, it was distributed under five prior selected health disorders groups and be compared and analyzed. Statistical significance was considered at $5 \%$ level of significance $(p=<0.05)$.

\section{RESULT AND ANALYSIS}

Total data is analysed to observe the difference in general w.r.t. the disorder group for Immunity.

The results are encouraging where Group - A Oil shows better results than Group - B Oil due to value addition through Vitamins.

\section{Immunity overall analysis}

In our study the serum IgG showed improvement in Group A (EBEVO1).The observed improvement in mean IgG due to consumption of EBEVO1 can elucidate the presence of Vitamin A, D E and $\mathrm{C}$ and omega 3 fatty acids. Role of Vitamin $\mathrm{E}$ and $\mathrm{C}$ as antioxidants also plays important role to reduced oxidative stress in body which can contribute to loss of immunity. Long chain omega-3 fatty acids may help to boost the immune system by enhancing the functioning of immune cells. More improvement can be observed for a longer period trial or consumption of EBEVO1 oil which is a daily consumable food. In Group B (EBEVO2) also slight changes are observed.

The total protein levels showed improvement in Group A (EBEVO1), where normal total protein levels are maintained. Statistically significant changes can only be observed and more improvement can be observed for a longer period trial or consumption of EBEVO1 oil, which is a daily consumable food. In Group B (EBEVO2) changes are observed

The albumin levels showed improvement in Group A (EBEVO1), where normal albumin levels are maintained. More improvement can be expected for a longer period consumption of EBEVO1 oil which is a daily consumable food. In Group B (EBEVO2) very slight changes are observed.

The serum globulin level showed improvement in Group A (EBEVO1). Since normal globulin levels considered to be within 2.0 to $3.5 \mathrm{~g} / \mathrm{dl}$, shows that it is maintained by the oil prescribed in group A (EBEVO1). More improvement can be observed for a longer period trial or consumption. In Group B (EBEVO2) also slight changes are observed showing the maintenance of globulin levels.

The hemoglobin levels showed improvement in Group A (EBEVO1), where normal hemoglobin levels are maintained. More improvement can be observed for a longer period trial or consumption of EBEVO1 oil which is a daily consumable food. In Group B (EBEVO2) very slight changes are observed.

The platelet count showed improvement in Group A (EBEVO1), where normal platelet counts were maintained. More improvement can be observed for a longer period trial or consumption of EBEVO1 oil that is a daily consumable food. In Group B (EBEVO2) negative changes are observed.

The handgrip tests left hand showed slight improvement in Group A (EBEVO1) where handgrip test left hand is maintained. More improvement can be observed for a longer period trial or consumption of EBEVO1 oil which is a daily consumable food. In Group B (EBEVO2) also shows slight changes.

The hand grip tests right hand showed slight improvement in Group A (EBEVO1)where hand grip test right hand is maintained. More improvement can be observed for a longer period trial or consumption of EBEVO1 oil which is a daily consumable food. In Group B (EBEVO2) also slight changes are observed.

\section{Immunity- disease wise data}

The Serum IgG showed improvement in Group A (EBEVO1). The observed improvement in mean IgG levels before and after consumption of EBEVO1 can elucidate the presence of anti-oxidants, Vitamin A, C, D and E. To copherol and to cotrienols actson the immunity levels. Long chain omega-3fatty acids may boost the immune system by enhancing the functioning of immune cells. Statistically significant changes can only be observed, and more improvement can be expected for a longer trial period, since EBEVO1 oil is a daily consumable food. In Group B (EBEVO2) also slight changes are observed.

The total protein levels showed improvement in Group A (EBEVO1), where normal total protein levels are maintained. Statistically significant changes can only be observed, and more improvement can be expected for a longer period trial, since EBEVO1 oil is a daily consumable food. In Group B (EBEVO2) changes are observed

The albumin levels showed improvement in Group A (EBEVO1), where normal albumin levels are maintained. Statistically significant changes can only be observed, and more improvement can be expected for a longer trial period, since EBEVO1 oil is a daily consumable food. In Group B (EBEVO2) very slight changes are observed.

The serum globulin level showed improvement in Group A (EBEVO1). Since normal globulin levels considered to be within 2.0 to $3.5 \mathrm{~g} / \mathrm{dl}$, shows that it is maintained by the oil prescribed in group A (EBEVO1). Statistically significant changes can only be observed, and more improvement can be expected for a longer trial period, since EBEVO1 oil is a daily consumable food. In Group B (EBEVO2) also slight changes are observed showing the maintenance of globulin levels.

The hemoglobin levels showed improvement in Group A (EBEVO1), where normal hemoglobin levels are maintained. Statistically significant changes can only be observed, and more improvement can be expected for a longer trial period, since EBEVO1 oil is a daily consumable food. In Group B (EBEVO2) very slight changes are observed.

The platelet count showed improvement in Group A (EBEVO1), where normal platelet counts are maintained. Statistically significant changes can only be observed, and more improvement can be expected for a longer trial period, since EBEVO1 oil is a daily consumable food. In Group B (EBEVO2) negative changes are observed.

The handgrip tests left hand showed slight improvement in Group A (EBEVO1) where handgrip test left hand is maintained. . Statistically significant changes can only be observed, and more improvement can be expected for a longer trial period, since EBEVO1 oil is a daily consumable food. In Group B (EBEVO2) also slight changes are observed.

The handgrip tests right hand showed slight improvement in Group A (EBEVO1) where handgrip test right hand is maintained. Statistically significant changes can only be observed, and more improvement can be expected for a longer trial period, since EBEVO1 oil is a daily consumable food. In Group B (EBEVO2) also slight changes are observed.

\section{DISCUSSION}


This study included 110 participants, divided into 2 groups after randomization. In group A 57 participants received the study oil (EBEVO1) and 53 participants in group B received sunflower oil (EBEVO2). The participants were provided oils for their whole family for 3 months. The participants were assessed with Clinical parameters (including both invasive and non-invasive)

The results showed that the test oil i.e. EBEVO1 was very effective on maintenance of albumin, globulin and hemoglobin. Favorable change was also observed on Serum Immunoglobulin. On the other hand, slight changes were found on the other oil (EBEVO2). It was observed that both oils maintained the clinical parameters.

Statistical significance compared in group A (EBEVO1) and group B $($ EBEVO2) showed significant changes in parameters such as total protein, albumin, hemoglobin and platelet count. Since both oils are daily consumable oils and not a medicine for any severe disorder. So improvements are seen on the Mean \pm SD changes of the parameters before and after consumption. Significant statistical changes are observed in only few parameters stated above.

EBEVO1 oil includes 5 vital nutrients such as Vitamin A, C, D, E and Omega 3 which are already proven scientifically in various trials worldwide. The special blend of added nutrients may improve metabolic functions to strengthen immunity to prevent general minor disorders or infections.

\section{CONCLUSION}

Vitamins have a role in the immune system, which extends to both innate and adaptive immune responses. Although some vitamins, such as vitamins $\mathrm{C}$ and $\mathrm{E}$ can act relatively in non specific manner in the immune system (as antioxidants), other vitamins, such as vitamins A and $\mathrm{D}$, can influence the immune response in highly specific ways. Vitamins A and D are notably distinct from other vitamins in that the irrespective bioactive metabolites, retinoic acid and Vitamin $\mathrm{D}_{2}-$ Ergocalciferol have hormone-like properties. Both of these metabolites are synthesized from their vitamin precursors by different tissues and cells in the body and exert their effects on target cells remotely by binding to nuclear-hormone receptors. Vitamin E, ascorbic acid (vitamin C) is one of the other essential nutrients, assisting in this antioxidant defense system by protecting against lipid peroxidation. In fact, vitamin $\mathrm{C}$ has been shown to function by sparing or reconstituting vitamin E, thus protecting lipid membrane. Although it is widely believed that the basic role of vitamin $\mathrm{E}$ is its function as an antioxidant, vitamin $\mathrm{C}$ has other important functions in numerous biological systems, including the synthesis of hormones, neurotransmitters, collagen and carnitine; the detoxification of exogenous compounds; and cytochrome P-450 activity. However, recent evidence has also shown that vitamins $\mathrm{E}$ and $\mathrm{C}$ are needed for normal function of the immune system.

\section{REFERENCE}

1. Mora, J Rodrigo et al "Vitamin effects on the immune system: vitamins A and D take centre stage." Nature reviews. Immunology vol. 8,9 (2008): 685-98.doi:10.1038/ nri 2378 .

2. Agarwal SC, Stout SD (28 June 2011). Bone Loss and Osteoporosis: An Anthropological Perspective. Springer Science \& Business Media. ISBN978-1-44198891-1.

3. EsraBirben,Oxidative Stress and Antioxidant Defense, World Allergy Organ J. 2012 Jan; 5(1):9-19

4. Saray Gutiérrez et al.Effects of Omega-3 Fatty Acids on Immune Cells,Int J Mol Sci. 2019 Oct; 20(20): 5028.

5. Bressani, R. \& Elias, L.G. (1974). Legume Foods. In "New Protein Foods. Vol. 1A. Technology" pp. 231-297. Altschul, A.M. ed.,Academic Press, NewYork.

6. Barbara Prietl;:Vitamin D and Immune Function; Nutrients. 2013; 5(7):2502-2521. 